\title{
Intracranial hemorrhage: ultrasound, CT and MRI findings
}

Huisman, Thierry A G M

\begin{abstract}
Intracranial hemorrhage is one of the most common causes of acute focal neurologic deficit in children and adults. Neuroimaging including ultrasonography (US), computer tomography (CT) and magnetic resonance imaging (MRI) is essential in the diagnosis of intracranial hemorrhage. Imaging findings should guide treatment. The highly variable appearance of an intracranial hemorrhage can be challenging. A thorough knowledge of hematoma evolution and US, CT and MR hematoma characteristics is mandatory for adequate interpretation of findings. The purpose of this review is (1) to summarize the imaging characteristics of intracranial hemorrhage on various imaging techniques and (2) to review the various types of intracranial hemorrhage, and their causes
\end{abstract}

DOI: https://doi.org/10.1007/s00330-004-2615-7

Posted at the Zurich Open Repository and Archive, University of Zurich

ZORA URL: https://doi.org/10.5167/uzh-156590

Journal Article

Published Version

Originally published at:

Huisman, Thierry A G M (2005). Intracranial hemorrhage: ultrasound, CT and MRI findings. European Radiology, 15(3):434-440.

DOI: https://doi.org/10.1007/s00330-004-2615-7 
Thierry A. G. M. Huisman

\section{Intracranial hemorrhage: ultrasound, CT and MRI findings}

Received: 12 July 2004

Accepted: 29 November 2004

Published online: 5 February 2005

(C) Springer-Verlag 2005

T. A. G. M. Huisman ( $\square)$

Department of Diagnostic Imaging, University Children's Hospital Zurich, Steinwiesstrasse 75 ,

8032 Zurich, Switzerland

e-mail: thierry.huisman@kispi.unizh.ch

Tel.: +41-1-2667110

Fax: +41-1-2667158

\begin{abstract}
Intracranial hemorrhage is one of the most common causes of acute focal neurologic deficit in children and adults. Neuroimaging including ultrasonography (US), computer tomography (CT) and magnetic resonance imaging (MRI) is essential in the diagnosis of intracranial hemorrhage. Imaging findings should guide treatment. The highly variable appearance of an intracranial hemorrhage can be challenging. A thorough knowledge of hematoma
\end{abstract}

evolution and US, CT and MR hematoma characteristics is mandatory for adequate interpretation of findings. The purpose of this review is (1) to summarize the imaging characteristics of intracranial hemorrhage on various imaging techniques and (2) to review the various types of intracranial hemorrhage, and their causes.

Keywords Intracranial hemorrhage · Ultrasonography $\cdot \mathrm{CT} \cdot \mathrm{MRI}$

\section{Introduction}

Intracranial hemorrhage is one of the most common causes of acute focal neurologic deficit in children and adults $[1,2]$. Acute morbidity and mortality, as well as final outcome, is influenced by an early and accurate diagnosis [1]. Imaging findings guide acute treatment and should prevent therapyinduced secondary complications. Computer tomography (CT), magnetic resonance imaging (MRI) and ultrasonography (US), mainly used in neonates, are well established imaging modalities in the diagnosis of intracranial hemorrhage [1-8]. The highly variable appearance of intracranial hemorrhage can, however, be challenging. Imaging characteristics depend on multiple factors including size and location of the hemorrhage, as well as biological factors such as the patient's hematocrit, tissue $\mathrm{pO}_{2}$ and $\mathrm{pH}$ and protein concentration (mainly hemoglobin, $\mathrm{Hb}$ ). In addition, the physiological breakdown of the hematoma with formation of various $\mathrm{Hb}$ derivatives (intracellular oxy- $\mathrm{Hb}$, intracellular deoxy-Hb, intracellular met-Hb, extracellular met- $\mathrm{Hb}$ and hemosiderin) will change the hematoma imaging characteristics over time [1-7]. Moreover, while ultrasonography and CT imaging characteristics of an intracranial hematoma are straightforward, the MRI charac- teristics are variable due to the complex interaction between the different hematoma breakdown products and the MRI used sequences [1-7]. Consequently, a thorough knowledge of hematoma evolution and MR signal characteristics is mandatory for adequate interpretation of MR findings.

Finally, US, CT and MRI findings can guide treatment by narrowing the differential diagnosis of intracranial hemorrhages.

The purpose of this review is (1) to summarize the imaging characteristics of intracranial hemorrhage on various imaging techniques and (2) to review the various types of intracranial hemorrhage, and their causes.

\section{Imaging characteristics of intracranial hemorrhage}

A basic knowledge of the pathophysiology and evolution of intracranial hemorrhages is essential for the understanding of the sequential imaging changes of intracranial hemorraghes.

Intracranial hemorhages are typically defined in five distinct stages: hyperacute ( $<12 \mathrm{~h})$, acute ( $12 \mathrm{~h}$ to 2 days), early subacute (2-7 days), late subacute ( 8 days to 1 month) and chronic ( $>1$ month to years) [3]. 
A hyperacute intracranial hemorrhage is initially liquid, composed of up to $98 \%$ oxygen saturated hemoglobin. Within the first hours, a complex heterogeneous mass consisting of a matrix of red blood cells (RBC), white blood cells (WBC) and platelet thrombi intermixed with protein-rich serum develops. Within the next few hours, the clot retracts. The expelled serum as well as a reactive vasogenic edema within the adjacent brain tissue, surrounds the retracting blood clot with densely packed RBCs. During the hyperacute stage, the trapped RBCs keep their normal biconcave shape and the incorporated hemoglobin remains oxygenated (intracellular $\mathrm{oxy}-\mathrm{Hb}$ ). With evolution to the acute stage, progressive $\mathrm{RBC}$ energy depletion results in dehydration and shrinking of the RBCs. At the same time, the intracellular hemoglobin becomes progressively deoxygenated (intracellular deoxy- $H b$ ). The cell membranes of the RBCs remain intact. In the subsequent early subacute phase, a centripetal oxidative denaturation of hemoglobin within the intact RBCs occur and deoxy$\mathrm{Hb}$ is consequently gradually converted to methemoglobin (intracellular meth- $\mathrm{Hb}$ ). During the following late subacute stage, progressing RBCs lysis result in a release of meth- $\mathrm{Hb}$ in the extracellular space (extracellular meth- $\mathrm{Hb}$ ). At the same time, the surrounding vasogenic edema slowly subsides. In the final chronic stage, macrophages and astroglial cells will surround the hematoma and slowly phagocytize the hematoma. Extracellular meth- $\mathrm{Hb}$ is converted by and stored within the macrophages as hemosiderin and ferritin. Eventually, the hematoma will resolve completely and a cystic fluid filled or collapsed brain defect will ensue. In adults, the hemosiderin/ferritin loaded macrophages can persist for years, while in children the macrophages may disappear with time [9].

Ultrasonography has traditionally been used in neonates, in whom the anterior fontanelle is used as an acoustic window to the cranial vault. The posterior and mastoid (posterolateral) fontanelles can be used for examining the posterior fossa, including cerebellum and brainstem [8]. This approach allows examination of the intracranial anatomy and disease processes at the bedside, and is cost-efficient. In addition, because of the lack of ionizing radiation and the fact that the examination can be performed without the need for sedation, neurosonography is exquisitely well suited for the detection of intracranial hemorrhages in neonates. One disadvantage is that ultrasonography is limited to neonates and small infants because the fontanelles typically close by 12 months of age [8]. Ultrasonography including color coded duplex sonography is done with phased-array, multiple-foci $5.0-$ or $7.0-\mathrm{MHz}$ transducers. The most frequent indication for neonatal ultrasonography is to rule out germinal matrix hemorrhage. In premature infants, intracranial hemorrhage is usually seen within the germinal matrix, the area along the ventricular wall from which the cortical neurones as well as glial cells originate. The germinal matrix is highly perfused because the neuronal proliferation is a very active metabolic pro- cess. Consequently, hemorrhages in premature infants typically occur within the germinal matrix (Fig. 1a, b, c). Ultrasonography has proven its value in the identification of germinal matrix hemorrhages and their complications (hydrocephalus). Germinal matrix hemorrhages are divided into four grades, depending upon their severity. Grade I is defined as a germinal matrix hemorrhage or subependymal hemorrhage with no or minimal intraventricluar extension. Grade II is a germinal matrix hemorrhage that extends into the normal sized ventricular system. Grade III is a germinal matrix hemorrhage that extends into the enlarged ventricular system. Grade IV is a germinal matrix hemorrhage that extends into the adjacent cerebral hemispheres. Hemispheric hemorrhage most probably results from a hemorrhagic venous ischemia (Fig. 1c) [12, 13]. Ultrasonography findings of germinal matrix hemorrhages parallel the previously described stages of intracranial hemorrhage; an exact differentiation between the stages is, however, limited. Ultrasonography shows germinal matrix hemorrhages as focal hyperechogenic lesions. On follow-up the hemorraghic clot retracts and becomes well defined and less echogenic. On late follow-up, the hematoma can dissolve completely with development of a cystic, fluid-filled brain defect. An intraventricular hemorrhage is usually easily delineated with a blood clot that is surrounded by hypoechogenic cerebrospinal fluid. On follow up a hyperechogenic lining of the ventricular system can persist for several weeks.

Subarachnoid blood is notoriously difficult to detect by ultrasonography, due to the wide normal variation in the ultrasound appearance of subarachnoid cisterns [8]. The identification of hematomas near the skull (epidural and subdural hematomas) is also limited because the bony edges of the fontanelles obscure the subdural or epidural spaces along the brain's surface.

Comparative studies have shown that $\mathrm{CT}$ and MRI have a better interobserver agreement for germinal matrix and intraventricular hemorrhage and reveal more instances of intraparenchymal hemorrhages compared with ultrasonography [14]. Nevertheless, ultrasonography remains an easy to perform, bed side, first line imaging tool in neonates.

Computer tomography represents the most frequently used imaging modality in the evaluation of hemorrhagic lesions in the acute setting $[1,2]$. CT is widely available and allows rapid and reliable visualization of the hemorrhage and of its possible complications [1,2]. Moreover, in most instances, CT provides important information about the etiology of the hemorrhage. Finally, the use of intravenous contrast agents offers the possibility of an angiographic display of the intracranial vasculature in the same examination.

CT imaging characteristics are determined by the degree of X-ray attenuation by the hemorrhage (Fig. 2a). The degree of blood clot retraction, the hematocrit, the hemoglobin fraction and protein content (globin) determine the density of the lesion on CT [9]. The appearance of an 

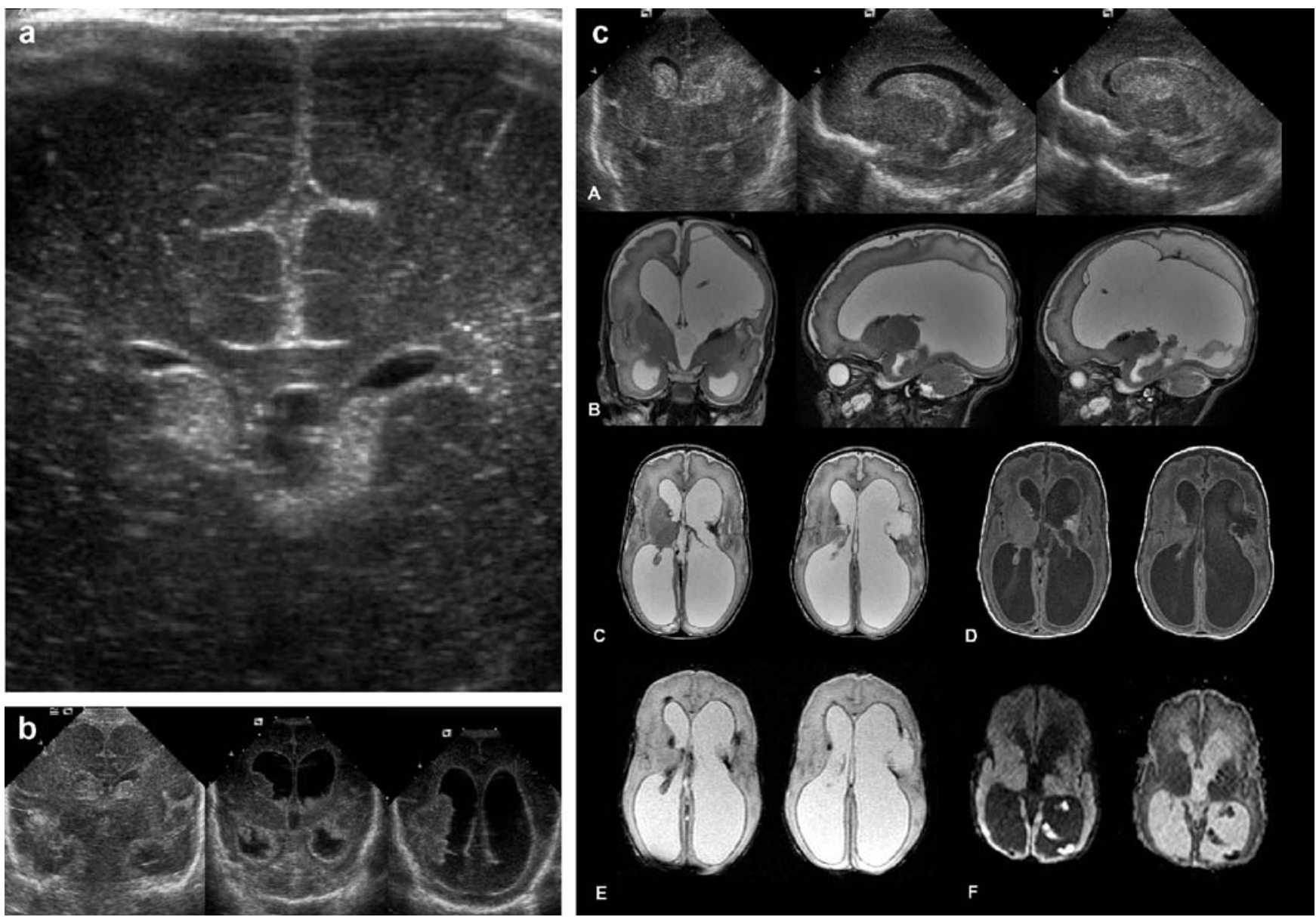

D
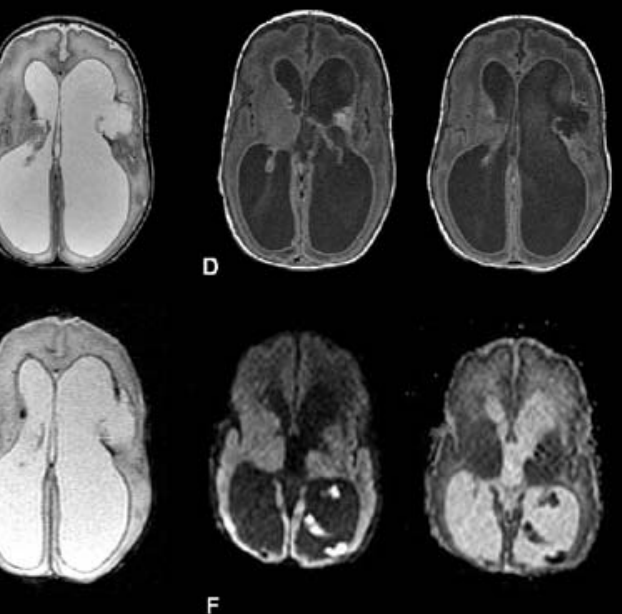

Fig. 1 a Cranial US in a 4-week-old premature (31 weeks gestation) girl demonstrating bilateral hyperechogenic subependymal hemorrhage grade I located at the foramen of Monroe. b Serial cranial US in a premature boy, gestational age: 9 weeks. The first US 3 days after birth, demonstrates a bilateral hyperechogenic subependymal hemorrhage grade I. On follow-up, the hemorrhage had progressed to a grade IV hemorrhage. Follow-up imaging (last two images) 13 days after birth, shows a retracting blood clot with a central hypoechogenic signal. In addition, a severe secondary hydrocephalus developed with hyperechogenic lining of the ventricular system due intraventricular extension of the hemorhage. c Serial US and MRI images of a premature boy, gestational age: 29 weeks. Ultrasonog-

raphy 5 days after birth (a) shows a grade I hemorrhage at the right side and a grade IV hemorrhage at the left side with extensive venous ischemia (hyperechogenic) within the periventricular white matter. Follow-up sagittal and axial T2-weighted MRI (b, c) 42 days after birth shows an extensive CSF filled porencephalic cyst at the left side as well as severe hydrocephalus. T2-hypointense blood products are seen subependymal at the basal ganglia. On T1-weighted MRI (d), the blood breakdown products are partially hyperintense. On T2* GRE MRI (e), the hemosiderin is seen best as hypointense signal alterations. On DWI and apparent diffusion coefficient (ADC) maps (f), the blood clots show a DWI-hyperintense and ADC-hypointense signal due to the blood clot retraction with restricted diffusion

intracranial hemorrhage varies on CT parallel to the temporal evolution of the hemorrhage. In the hyperacute phase, the matrix mass of RBCs, WBCs, platelets and interspersed serum is equally dense as the adjacent normal brain parenchyma. Consequently, in the hyperacute phase it can be difficult to delineate the hemorrhage. In the acute and early subacute phase, progressive blood clot retraction with concomitant extrusion of the serum component will rapidly increase the density of the hemorrhage. In particular, the increasing concentration of the protein component of hemoglobin (globin) will appear hyperdense on CT. Simultaneously a hypodense rim develops around the hemor-

rhage due to serum extrusion and reactive perifocal vasogenic edema. During the subsequent late subacute phase, progressive RBC lysis and proteolysis of the globin protein result in decreasing density of the hematoma. In the late subacute phase the hematoma may become isodense to the adjacent brain parenchyma (Fig. 2b). The injection of contrast media can be helpful in the delineation of these isodense hematomas because the periphery of the hematoma may show enhancement due to a disrupted blood-brain barrier [1]. During the chronic phase, the hypodense hematoma will be progressively phagocytized, ultimately resulting in a resolution of the hemorrhage. Typically a small 
Fig. 2 a Serial axial CT and coronal T2-weighted MRI in a 22 -year-old male with a frontobasal contusion. CT in the early subacute phase shows a centrally hyperdense blood clot surrounded by a hypodense rim of vasogenic edema and extrused serum. Follow-up coronal MRI in the chronic phase shows a T2-hyperintense CSF filled brain defect lined by a T2hypointense rim of hemosiderin. b Axial pre- and post-contrast $\mathrm{CT}$ in a 54-year-old male with a chronic right $\mathrm{SDH}$. The $\mathrm{SDH}$ is nearly isodense to the adjacent cortex. After injection of contrast agent, the displaced contrast enhancing cortical vessels facilitate lesion detection a

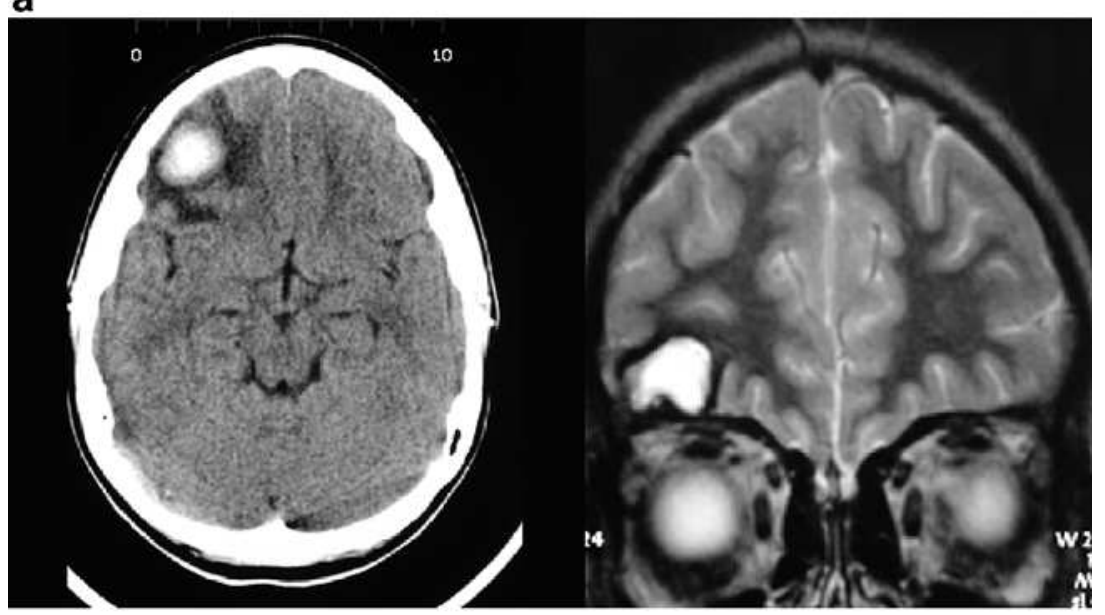

b

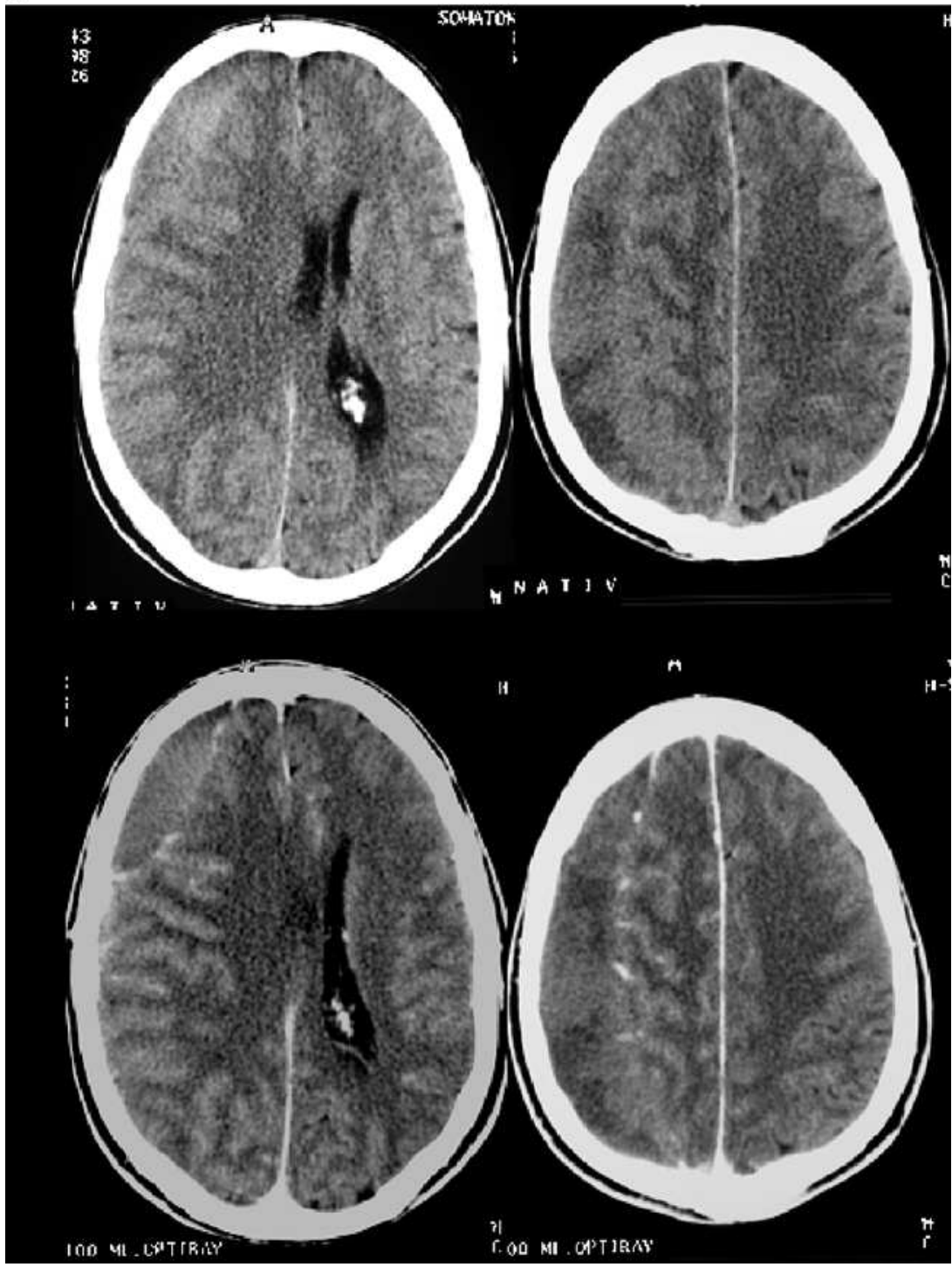


hypodense brain defect remains. Occasionally a focal enlargement of neighbouring sulci or a focal atrophy as well as residual hyperdense calcifications remain $[1,9]$.

In cases of severe anemia (low hematocrit), due to the reduced concentration of the globin protein, the acute hematoma will be less hyperdense than expected. In addition, rebleeding within a chronic hematoma will increase the overall density of the hematoma, which can be misleading in the estimation of the age of the hemorrhage.

Extra-axial hemorrhages (epidural and subdural hematomas) parallel the intraparenchymal hematomas in their $\mathrm{CT}$ appearance. Acute subarachnoid hemorrhages (SAH) are also hyperdense. The density of the SAH decreases rapidly, however, due to the dilution by cerebrospinal fluid.

Magnetic resonance imaging is widely accepted as the most sensitive imaging modality for intracranial hemorrhage and its sequelae. The temporal evolution of an intracranial hematoma can be studied in detail, and follows the previously described sequence. The selective sensitivity of the different MRI sequences combined with MRI sequence related varying signal intensities of an intracranial hemorrhage increase diagnostic accuracy significantly. MR signal characteristics are determined by the paramagnetic effects of the different breakdown products of hemoglobin, the magnetic field strength, the used pulse sequence and many other technical factors $[1,2]$. Intracellular oxy- $\mathrm{Hb}$, intracellular deoxy- $\mathrm{Hb}$, intracellular met- $\mathrm{Hb}$, extracellular met- $\mathrm{Hb}$ and hemosiderin have different magnetic properties due to the different oxidation state of iron as it is incorporated or released from the hemoglobin macromolecule. Depending on the number of unpaired electrons in the outer orbit of the iron atom, these hematoma breakdown products will be diamagnetic (oxy- $\mathrm{Hb}$, no unpaired electrons), paramagnetic (deoxy- $\mathrm{Hb}$ and met- $\mathrm{Hb}$, few unpaired electrons) or superparamagnetic (hemosiderin, many unpaired electrons). Consequently in the hyperacute stage the hemorrhage is T1-iso or hypointense and T2 hyperintense; in the acute stage T1-iso or hypointense and T2-hypointense, in the early subacute stage T1hyperintense and T2-hypointense, in the late subacute stage $\mathrm{T} 1$ and T2-hyperintense and finally in the chronic phase T1hypointense and T2-centrally hyperintense, surrounded by a rim of hypointensity (hemosiderin) (Fig. 2a). T1- and T2-weighted MR sequences are classically used for delineation of the extent and estimation of the age of an intracranial hemorrhage. The age of a lesion is of diagnostic importance because it determines the management of the patient. Acute and chronic hemorrhages require different treatment plans. Many other MR sequences have been developed to increase the diagnostic accuracy of MR imaging. Fluid attenuated inversion recovery (FLAIR) and T2*-gradient and echo (T2*-GRE) MR sequences have been applied in the depiction of intracranial hemorrhages. The high sensitivity of T2*-GRE MR sequences (Fig. 1c) for the susceptibility effects of paramagnetic and superparamagnetic substances increases the number of hemorrhagic lesions that can be identified [3, 6, 7]. A major limitation of the T2*-GRE sequence is that this sequence cannot help in estimating the age of the hematomas [3] FLAIR sequences are favourable in the detection of SAH because the suppression of the cerebrospinal fluid signal in combination with the strong T2-weighting enhances the appearance of small amounts of blood within the cerebrospinal fluid of the ventricles or extra-axial spaces (Fig. 3) $[1,7]$. A recent study showed, however, that FLAIR sequences do not provide any additional information than T2-weighted sequences for intraparenchymal hemorrhages [3]. The value of diffusion weighted imaging (DWI) in the evaluation of intracranial hemorrhage is still under active investigation $[4,6,7]$. DWI seems to be promising in differentiating primary intracerebral hemorrhage from hemorrhagic cerebral ischemia [4].
Fig 3 Axial FLAIR images in a 24-year-old male with an acute small SDH. The suppression of the CSF signal enhances detection of the frontal FLAIR-hyperintense SDH

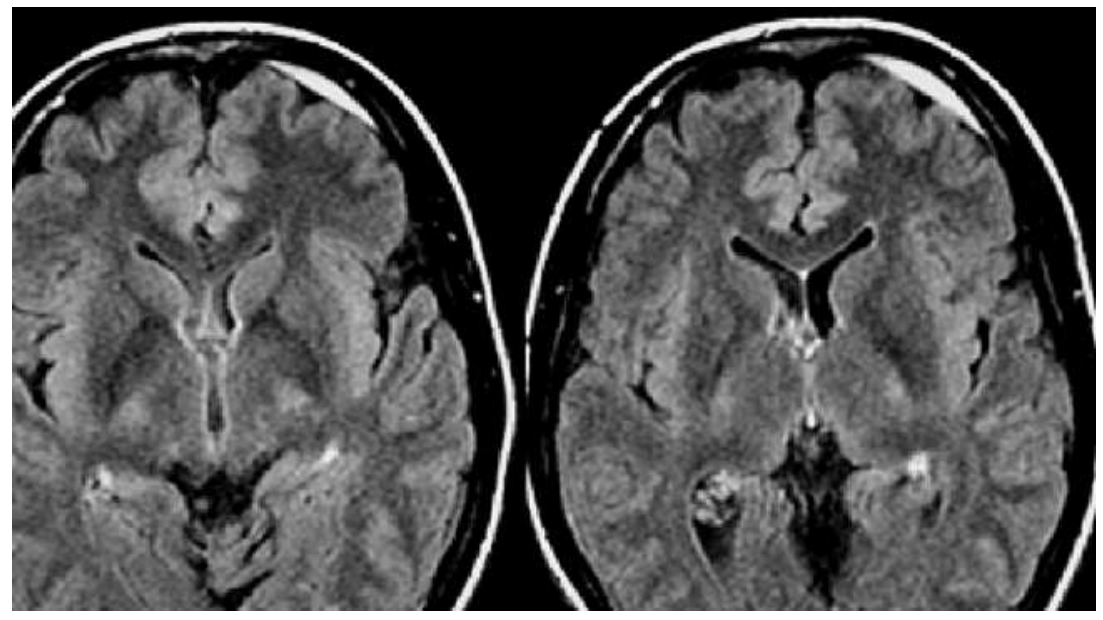




\section{Various types of intracranial hemorrhage and their cause}

Intracranial hemorrhage can be classified according to their location and etiology. Location and etiology are tightly coupled. An epidural hematoma results, for example, most frequently from a traumatic skull fracture, while a subarachnoid hemorrhage usually results from a ruptured aneurysm. Consequently, the neuroradiologist should analyze and locate the hemorrhage in detail to get information about the possible etiology of the hemorrhage to guide treatment.

Intracranial hemorrhages can be (1) epidural, (2) subdural, (3) subarachnoidal, (4) intraventricular, (5) intracerebral or (6) may involve a combination of multiple compartments.

The etiologic factors for intracranial hemorrhage are numerous. Among the main categories are hemorrhagic stroke, arterial hypertension, head trauma, aneurysms and vascular malformations, intracranial neoplasms, hematologic disorders, drugs, cerebral amyloid angiopathy, angiitis and miscellaneous angiopathies $[10,11]$.

Epidural hematomas (EDH) usually occur in the setting of acute head traumas. Clinical presentation may be delayed (symptom free or "lucid" interval). Arterial EDH usually results from a laceration of branches of the meningeal arteries within the skull, while venous EDH result from a tear of dural venous sinuses. Venous EDH are more frequently encountered in children. Venous EDH may develop more slowly and the clinical symptoms are less acute than arterial EDH. A rapidly enlarging arterial EDH can result in a significant midline shift with different degrees of secondary herniation and possible ischemia. EDH are typically well-defined biconvex extracerebral lesions that may displace the adjacent brain. EDH are located between the skull and the periostal layer of the dura mater. The tight adherence between the dura mater and the skull prevents extension of an EDH across skull sutures [15]. $\mathrm{CT}$ attenuation and MR signal intensities follow the previously described temporal evolution. No dilution occurs with cerebrospinal fluid because an EDH is separated from the CSF by the dura mater.

Subdural hematomas (SDH) can be of traumatic or nontraumatic origin. In SDH, blood is accumulated between the dura mater and the arachnoidea. SDH are usually of venous origin and result from lacerations of cortical veins that cross the subarachnoid space before entering the dural sinuses. A concomitant arachnoidal laceration with leakage of CSF from the subarachnoid space into the subdural space results in a mixture of CSF and venous blood. SDH may occur spontaneously in elderly patients. They usually enlarge slowly with initially minimal neurologic symptoms, and may evolve to larger chronic hematomas that compress the adjacent brain. In children, SDHs are commonly seen in cases of child abuse. SDHs are typically crescent-shaped along the surface of the brain or along dural duplications such as the falx cerebri and tentorium cerebelli. Because they are located between the dura mater and the arachnoidea, SDHs may cross skull sutures. The CT attenuation or MR signal intensities also follow the previously described temporal evolution. In cases of a significant mixture with CSF the progression of blood clot resorption can be accelerated $[1,2]$.

Subarachnoid hemorrhage (SAH) can be of traumatic and non-traumatic origin. In SAH, blood is accumulated within the subarachnoid space, that is the space between the arachnoidea and the pia mater. SAH can occur in head injury due to laceration of cortical veins or arteries that course in the subarachnoid space but may also occur due to cortical contusions and lacerations with extravasation of blood into the subarachnoid space. A typical location for $\mathrm{SAH}$ after head trauma is within the interpeduncular cistern or in the sylvian fissure [15]. Spontaneous SAH usually result from ruptured aneursyms (older patients) or arteriovenous vascular malformations (younger patients). CT is the preferred method of imaging SAH because it is very sensitive in detecting blood in the CSF space and is usually more easily available in the acute setting of a SAH. Typically, in acute SAH hyperdense blood is seen within the basal cisterns and subarachnoid spaces. The location and distribution of the SAH can indicate the site of the aneurysm. In addition, contrast enhanced CT angiography can display the exact location and shape of the aneurysm. On MRI, the rapid dilution of the hemorrhage by CSF can hamper the detection of a SAH. FLAIR sequences have shown to be very sensitive in the detection of acute SAH because the suppression of the CSF signal enhances the conspicuity of SAH [1, 2]. Identification of SAH by neonatal ultrasonography is very limited due to the wide normal variation in the normal ultrasound appearance of subarachnoid cisterns [8].

Intraventricular hemorrhage (IVH) can result from the secondary extension of a neonatal germinal matrix hemorrhage into the ventricular system as previously described or from the reflux of blood from the subarachnoid spaces into the ventricular system after spontaneous or traumatic SAH. In addition, an intraparenchymal hemorrhage may rupture into the ventricular system. Traumatic IVH is rare and usually associated with other manifestations of intracranial posttraumatic lesions [9]. In analogy to SAH, the blood is rapidly diluted by the CSF. Ultrasonography in neonates and CT in adults are the primary, acute imaging modalities. MRI, especially FLAIR sequences, can also be helpful in the detection of IVH. Fluid sedimentation levels are frequently seen within the dependant parts of the ventricular system. On late follow up MRI, a T2-hypointense lining of the ventricular system can persist due to hemosiderin depositions.

Intracerebral hemorrhages (ICH) has many different etiologies which can roughly be divided into traumatic and spontaneous reasons. Post-traumatic ICH are relatively straightforward and result from the direct impact of forces to the skull and underlying brain with laceration and rup- 
ture of intraparenchymal vessels. Arterial hypertension, hemorrhagic stroke and coagulation disorders including anticoagulant drugs are among the most frequent reasons for spontaneous ICH. In addition, spontaneous ICH can occur due to the rupture of intracranial aneurysms and vascular malformations as well as due to hemorrhages within intracerebral neoplasms. The shape and imaging characteristics of an ICH follow the previously described evolution characteristics of a hematoma. US can easily identify intraparenchymal hemorrhages in neonates, CT and MRI are both very sensitive in the detection of ICH. The multiplanar imaging capability and the different MR imaging sequences (T1, T2, T2*, FLAIR, DWI) can increase the diagnostic accuracy of MRI compared to CT. One disadvantage is that the monitoring of a critically diseased patient is somewhat limited in MRI compared with CT.
Combinations of the different kinds of intracranial hemorrhages are usually encountered in critically injured trauma patients. Prognosis is usually limited.

\section{Conclusion}

A proper knowledge of the imaging characteristics of intracranial hemorrhages and their evolution over time as well as an insight into the different etiologies of intracranial hemorrhages are mandatory to guide treatment. US, CT and MRI rely on different techniques. The neuroradiologist should be aware of the advantages and disadvantages of each individual imaging technique. US is almost exclusively limited to neonatal hemorrhages, while CT is most frequently used in the acute setting. MRI is used when the CT imaging findings do not explain the neurologic symptoms or if a discrepancy exists between the CT imaging findings and neurology.

\section{References}

1. Parizel PM, Makkat S, Van Miert E, van Goethem JW, van den Hauwe L, De Schepper AM (2001) Intracranial hemorrhage: principles of CT and MRI interpretation. Eur Radiol 11:17701783

2. Anzalone N, Scotti R, Riva R (2004) Neuroradiologic differential diagnosis of cerebral intraparenchymal hemorrhage. Neurol Sci 24:S3-S5

3. Alemany Ripoll M, Stenborg A, Sonninen P, Terent A, Raininko R (2004) Detection and appearance of intraparencymal haematomas of the brain at $1.5 \mathrm{~T}$ with spin-echo, FLAIR and GE sequences: poor relationship to the age of the haematoma. Neuroradiology 46:435-443

4. Zaheer A, Ozsunar Y, Schaefer PW (2000) Magnetic resonance imaging of cerebral hemorrhagic stroke. Top Magn Reson Imaging 11(5):288-299
5. Wasenko JJ, Lieberman KA, Rodziewicz GS, Holsapple (2002) Magnetic resonance imaging characteristics of hyperacute hemorrhage in the brain and spine. Clin Imaging 26:330-337

6. Wiesmann M, Mayer TE, Yousry I, Hamann GF, Brückmann H (2001) detection of hyperacute parenchyma hemorrhage of the brain using echoplanar $\mathrm{T} 2 *$-weighted and diffusionweighted MRI. Eur Radiol 11:849-853

7. Lin DDM, Filippi CG, Steever AB, Zimmerman RD (2001) Detection of intracranial hemorrhage: comparison between gradient-echo images and $b_{0}$ images obtained from diffusionweighted echo-planar sequences. Am J Neuroradiol 22:1275-1281

8. Di Salvo DN (2001) A new view of the neonatal brain: clinical utility of supplemental neurologic US imaging windows. Radiographics 21:943-955

9. Osborn AG (1994) Intracranial hemorrhage. In: Diagnostic neuroradiology. Mosby, St Louis, pp 154-198

10. Garcia JH (1997) Vascular diseases. In: Neuropathology the diagnostic approach. Mosby, St Louis, pp 263-320
11. Hillemans A, Kortmann RD, Herrlinger U, Skalej M, Krapf H (2003) Recurrent delayed brain hemorrhage over years after irradiation and chemotherapy for astrocytoma. Eur Radiol 13(8):18911894

12. Volpe JJ (2000) Intracranial haemorrhage: subdural, primary subarachnoid, intracerebellar, intraventricular (term infant), and miscellaneous. In: Volpe JJ (ed) Neurology of the newborn. Intracranial hemorrhage, 4th edn. Saunders, Philadelphia, pp 397-429

13. Barkovich AJ (1995) Destructive brain disorders of childhood. In: Barkovich AJ (ed) Pediatric neuroimaging. 2nd edn. Raven Press, New York, pp 107 175

14. Blankenberg FG, Loh NN, Bracci P et al (2000) Sonography, CT, and MR imaging: a prospective comparison of neonates with suspected intracranial ischemia and hemorrhage. Am J Neuroradiol 21:213-218

15. Gean AD (1994) Extra-axial collections. In: Imaging of head trauma. Raven Press, New York, pp 75-145 